\title{
ALGEBRAS STABLY EQUIVALENT TO NAKAYAMA ALGEBRAS OF LOEWY LENGTH AT MOST 4
}

\author{
IDUN REITEN \\ (Received 1 February; revised 29 May 1978) \\ Communicated by $\mathbf{H}$. Lausch
}

\begin{abstract}
Two artin algebras $\Lambda$ and $\Lambda^{\prime}$ are said to be stably equivalent if their categories of finitely generated modules modulo projectives are equivalent. In this paper a characterization is given of the artin algebras stably equivalent to Nakayama algebras of Loewy length (at most) four. The proof is an illustration of the technique of using irreducible maps to study problems about stable equivalence.
\end{abstract}

Subject classification (Amer. Math. Soc. (MOS) 1970): 16 A 46, 16 A 64.

\section{Introduction}

The purpose of this note is to give a classification of the artin algebras stably equivalent to a Nakayama algebra of Loewy length at most 4 , as was announced in Reiten (1977). Before we state our main result, we shall recall some definitions.

An artin algebra $\Lambda$ is Nakayama (that is, generalized uniserial) if each indecomposable left and right projective $\Lambda$-module has a unique composition series. Let $\bmod \Lambda$ denote the category of finitely generated left $\Lambda$ modules. Two artin algebras $\Lambda$ and $\Lambda^{\prime}$ are said to be stably equivalent if the categories $\bmod \Lambda$ and $\bmod \Lambda^{\prime} \operatorname{modulo}$ projectives are equivalent categories. Here the objects of $\overline{\bmod \Lambda}$ are the same as those of $\bmod \Lambda$, denoted by $\underline{M}$ for an $M$ in $\bmod \Lambda$, and $\operatorname{Hom}(\underline{M}, \underline{N})=\operatorname{Hom}_{\Lambda}(M, N)$ / $P(M, N)$, where $P(M, N)$ is the subgroup whose elements are the $f: M \rightarrow N$ which factor through a projective $\Lambda$-module. We denote the image of $f$ in $\operatorname{Hom}(\underline{M}, \underline{N})$ by $\underline{f}$. We further let $\mathbf{r}$ denote the radical of $\Lambda$ and we denote by $L L(M)$ the Loewy length of a $\Lambda$-module $M$, that is, the smallest $i \geqslant 0$ such that $\mathbf{r}^{t} M=0$.

We are now ready to state our classification theorem. We point out that we know from Reiten (1975) that if $\Lambda$ is stably equivalent to a Nakayama algebra of Loewy length at most 3 , then $\Lambda$ is also Nakayama. 
THEOREM. An indecomposable non-Nakayama algebra $\Lambda$ is stably equivalent to a Nakayama algebra $\Lambda^{\prime}$ of Loewy length (at most) 4 if and only if the following conditions hold.

The indecomposable projective (injective) $\Lambda$-modules which are not uniserial have Loewy length 3, length 4 and simple socle.

The simple $\Lambda$-modules $T_{1}, T_{2}, \ldots, T_{n}, T_{n+1}=T_{1}, \ldots, n \geqslant 3$, can be ordered in such a way that no two of the $T_{1}, \ldots, T_{n}$ are isomorphic, and such that the following holds.

If $P_{i}$, the projective cover of $T_{i}$, is not uniserial, then $\operatorname{soc} P_{i}=T_{i+3}, \mathbf{r} P_{i} / \mathbf{r}^{2} P_{i} \cong$ $T_{i+1} \amalg T_{i+2}, P_{i+1}$ is uniserial of length $3, \operatorname{soc} P_{i+1}=T_{i+4}, P_{i+2}$ is uniserial of length 2 or 3. If $P_{t+2}$ has length 3 , then soc $P_{t+2}=T_{t+5}$ and $P_{t+3}$ is not uniserial. If $P_{t+2}$ has length 2, then $P_{i+3}$ is not uniserial or is uniserial of length 3.

If $P_{i}$ is uniserial of length 3 and $T_{t}$ is not a summand of any $\mathbf{r} P_{j} / \mathbf{r}^{2} P_{j}$ for $P_{j}$ not uniserial, then $T_{t+1}=\mathbf{r} P_{i} / \mathbf{r}^{2} P_{i}$, and $P_{t+1}$ is uniserial of length 3 or is not uniserial.

This result contributes to the general problem of understanding what it means for two artin algebras to be stably equivalent. It is known that if $\Lambda$ is stably equivalent to a self injective algebra, then each indecomposable ring summand of $\Lambda$ which has Loewy length at least 3 is also self injective (Reiten, 1976). Further, a description is known of the artin algebras stably equivalent to hereditary algebras (Auslander and Reiten (1973, 1975)), and Gabriel and Riedtmann have studied artin algebras stably equivalent to self injective Nakayama algebras over an algebraically closed field.

An important problem in the theory of stable equivalence is whether two stably equivalent algebras have the same number of nonprojective simple modules. In view of the fact that we know that this is the case if one of the algebras is Nakayama (see Reiten, 1978 for a proof of this and a list of other cases where it is known), it is of interest also in connection with this problem to describe the algebras stably equivalent to a Nakayama algebra.

Our proof is also an illustration of the technique of using the irreducible maps introduced in Auslander and Reiten (1977a) to study problems about stable equivalence. We shall devote Section 1 to explaining how we use irreducible maps in this paper together with recalling the necessary background material. It is essentially the method we used in Reiten (1975), which we shall here present from a different point of view. Section 2 is devoted to proving that the conditions are necessary, and Section 3 to proving that they are sufficient.

\section{Background and method}

Let $\Lambda$ be an artin algebra. We recall that a $\operatorname{map} f: A \rightarrow B$ in $\bmod \Lambda$ is said to be irreducible if $f$ is neither a split monomorphism nor a split epimorphism, and given any commutative diagram 


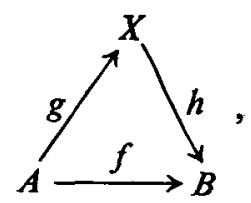

then $g$ is a split monomorphism or $h$ is a split epimorphism (Auslander and Reiten, 1977a). Assume that $A$ and $B$ are indecomposable and not projective. Then we know that $f: A \rightarrow B$ is irreducible if and only if $\underline{f}: \underline{A} \rightarrow \underline{B}$ is irreducible (Auslander and Reiten, 1977b).

We associate a diagram $\mathrm{D}$ with $\Lambda$, consisting of the indecomposable objects in $\bmod \Lambda$, together with an arrow $A \rightarrow B$ from $A$ to $B$ if there is an irreducible map from $A$ to $B$, for $A$ and $B$ indecomposable in $\bmod \Lambda$. For an indecomposable nonprojective $C$ in $\bmod \Lambda$, we shall denote by $\mathrm{D}(C)$ the part of the diagram where the objects are $C$ and the indecomposable nonprojective objects $X$ such that there is some composition of irreducible maps from $X$ to $C$ which is not zero modulo projectives, and with an arrow $A \rightarrow B$ when there is an irreducible map from $A$ to $B$, for objects $A$ and $B$ in $\mathrm{D}(C)$. If we know that there is some nonzero composition of irreducible maps from $A$ to $B$ modulo projectives in $\mathrm{D}(C)$, without knowing if there is actually some irreducible map from $A$ to $B$, we shall indicate this by writing a dotted arrow $A$........ $B$.

An equivalence $F: \underline{\bmod \Lambda} \rightarrow \bmod \Lambda^{\prime}$ induces a one-one correspondence between the indecomposable nonprojective objects of $\bmod \Lambda$ and $\bmod \Lambda^{\prime}$. If $\Lambda$ and $\Lambda^{\prime}$ are stably equivalent and $C$ and $C^{\prime}$ correspond to each other, then we must have that the diagrams $\mathrm{D}(C)$ and $\mathrm{D}\left(C^{\prime}\right)$ correspond to each other. This fact will be the main basis for our method.

We also recall that the category $\bmod \Lambda \operatorname{modulo}$ injectives, denoted $\overline{\bmod \Lambda}$, is equivalent to $\bmod \Lambda$ (Auslander and Reiten, 1973). If $A$ and $B$ are indecomposable noninjective modules, then $f: A \rightarrow B$ is irreducible if and only if $\bar{f}: \bar{A} \rightarrow \bar{B}$ is irreducible (Auslander and Reiten, 1977b). Here the objects in $\overline{\bmod \Lambda}$ are denoted by $X$ for $X$ in $\bmod \Lambda$, and $\bar{f}: \bar{A} \rightarrow \bar{B}$ denotes the image of $f$ in $\operatorname{Hom}(\bar{A}, \bar{B})=\operatorname{Hom}_{\Lambda}(A, B) / I(A, B)$, where $I(A, B$ consists of the maps from $A$ to $B$ which factor through an injective module. We shall denote by $\mathrm{D}^{\prime}(C)$ the diagram belonging to an indecomposable noninjective module defined in an analogous way to $\mathrm{D}(C)$.

Let now $\Lambda^{\prime}$ be an indecomposable Nakayama algebra. It is well known that for every indecomposable $\Lambda^{\prime}$-module $C, C / \mathrm{r} C$ is simple. It is then not hard to see (Auslander and Reiten, 1977a, Section 4) that if $A$ and $B$ are indecomposable in $\bmod \Lambda$, then $f: A \rightarrow B$ is irreducible if and only if $f$ is an epimorphism with $\operatorname{Ker} f$ simple or $f$ is a monomorphism with $\operatorname{Im} f$ a maximal submodule of $B$.

For a Nakayama algebra $\Lambda^{\prime}$ of Loewy length 4 , we then have the following diagrams associated with the various types of indecomposable nonprojective $\Lambda^{\prime}$-modules, where we assume that $\Lambda^{\prime}$ is not local. 
(1) Let $P$ be an indecomposable projective $\Lambda^{\prime}$-module of length 4 . Then we have

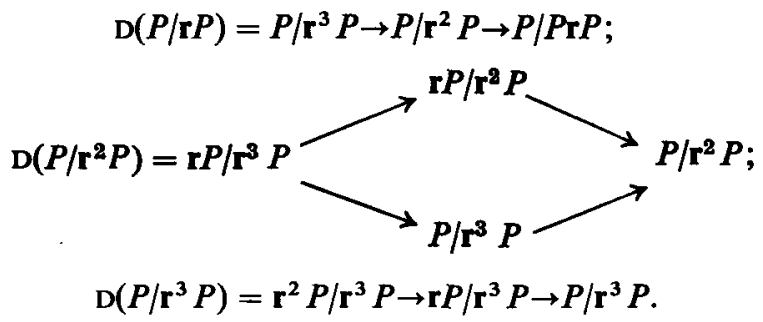

(2) If $P$ is indecomposable projective of length 3 , we have

$$
\begin{aligned}
& \mathrm{D}(P / \mathbf{r} P)=P / \mathbf{r}^{2} P \rightarrow P / \mathbf{r} P ; \\
& \mathrm{D}(P / \mathbf{r} P)=\mathbf{r} P / \mathbf{r}^{2} P \rightarrow P / \mathbf{r}^{2} P .
\end{aligned}
$$

(3) If $P$ is indecomposable projective of length 2 , we have

$$
\mathrm{D}(P / \mathbf{r} P)=P / \mathbf{r} P \text {. }
$$

We also point out that if $A$ and $B$ are indecomposable nonprojective $\Lambda^{\prime}$ modules, and $\underline{f}: \underline{A} \rightarrow \underline{B}$ and $\underline{g}: \underline{A} \rightarrow \underline{B}$ are not zero, there is some $t: A \rightarrow A$ such that $\underline{f} \underline{t}=\underline{g}$ or $\underline{g} \underline{t}=\underline{f}$ and some $s: B \rightarrow B$ such that $\underline{s} \underline{f}=\underline{g}$ or $\underline{s} \underline{g}=\underline{f}$. We finally point out that we shall often use the easily verified fact that if $f: A \rightarrow B$ is an epimorphism in $\bmod \Lambda^{\prime}$ and $A$ and $B$ are indecomposable and not projective, then $: \underline{f} \rightarrow \underline{B}$ is not 2 zero.

\section{Necessity}

Throughout this section let $\Lambda$ be an indecomposable artin algebra stably equivalent to a Nakayama algebra $\Lambda^{\prime}$ of Loewy length 4 . In this section we shall prove that the conditions stated in the Theorem are necessary.

We denote the simple $\Lambda$-modules by $T_{t}$ and their projective covers by $P_{l} . X=$ $\left(\begin{array}{l}T_{1} \\ T_{2}\end{array}\right)$ denotes a uniserial module of length 2 with $X / \mathbf{r} X \cong T_{1}, \operatorname{soc} X \cong T_{2}$. Similarly, $X=\left(\begin{array}{l}T_{1} \\ T_{2} \\ T_{3}\end{array}\right)$ denotes a uniserial module of length 3 with $X / \mathrm{r} X \approx T_{1}$, soc $X \cong T_{3}$ and $\mathbf{r} X / \mathbf{r}^{2} X \cong T_{2}$. It will follow from Lemma 1 that $X$ is uniquely determined if it exists.

We denote the simple $\Lambda^{\prime}$-modules by $S_{t}$ and their projective covers by $Q_{t}$, in such a way that if $S_{i}$ is not projective, then $\mathbf{r} P_{i} / \mathbf{r}^{2} P_{i} \cong S_{i+1}$. We consider a fixed stable equivalence between $\bmod \Lambda$ and $\bmod \Lambda^{\prime}$, and denote by $\leftrightarrow$ the induced correspondence between the indecomposable nonprojective modules over $\Lambda$ and $\Lambda^{\prime}$.

We start out with the following result which is also proved in Reiten (1975), but we include a proof here for the sake of completeness. Here $L$ denotes the length of a module. 
Lemma 1. Let $P$ be an indecomposable projective $\Lambda$-module.

(a) $L(P) \leqslant 4$ and so if $L L(P)=4$, then $P$ is uniserial.

(b) If $L L(P)=3$, then $\mathbf{r}^{2} P$ is simple and $\mathbf{r} P / \mathbf{r}^{2} P$ is either simple or the direct sum of two nonisomorphic simple modules.

(c) If $L L(P)=2$, then $P$ is uniserial.

(d) If $I$ is an indecomposable injective $\Lambda$-module which is not uniserial, then $L L(I)=3, I / \mathbf{r} I$ is simple and $\mathbf{r} I / \mathbf{r}^{2} I$ is either simple or the direct sum of two nonisomorphic simple modules.

(e) The indecomposable projective (injective) $\Lambda$-modules which are not uniserial of length 2 are injective (projective).

Proof. (a) Assume $L(P) \geqslant 5$, and choose a chain of submodules with proper inclusions $(0)=P_{0} \subset P_{1} \subset P_{2} \subset P_{3} \subset P_{4} \subset P$. Then $P / P_{1} \ldots \ldots . \cdots P / P_{2} \ldots \ldots . \cdots P / P_{3}$ ........ $P / P_{4}$ would be part of $\mathrm{D}\left(P / P_{4}\right)$, and this is a contradiction.

(b) If $L L(P)=3$, we have by (a) that $L\left(\mathbf{r}^{2} P\right) \leqslant 2$ and $L\left(\mathbf{r} P / \mathbf{r}^{2} P\right) \leqslant 2$. Assume that $\mathbf{r}^{2} P=T_{1}\left\lfloor T_{2}\right.$. If $T_{1} \cong T_{2}$, we would have a commutative diagram

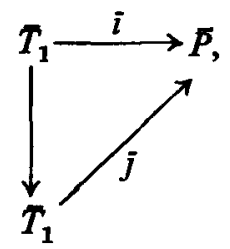

where $i: T_{1} \rightarrow P$ and $j: T_{1} \rightarrow P$ are inclusion maps, but this is a contradiction. Since then $T_{1}$ and $T_{2}$ are not isomorphic, we have that

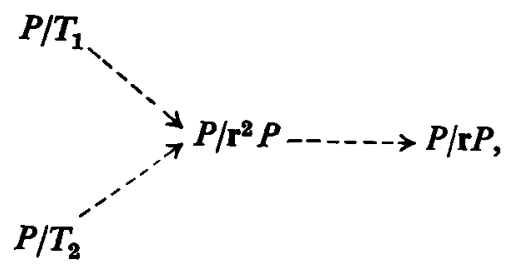

is part of $\mathrm{D}(P / \mathbf{r} P)$, which is impossible. Hence $L\left(\mathbf{r}^{2} P\right)=1$.

(c) Assume $L L(P)=2$. By (a) we know that $L(\mathrm{r} P) \leqslant 3$. As in (b), we can show that $\mathbf{r} P$ has no repeated summands. Assume first $L(\mathbf{r} P)=3$, and write

$$
\mathbf{r} P=T_{1} \bigsqcup T_{2} \bigsqcup T_{3} .
$$

We get a contradiction by considering the following part of $\mathrm{D}^{\prime}(P)$.

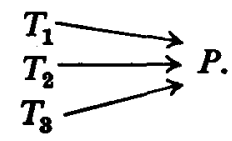


Assume then $L(\mathbf{r} P)=2$, and write $\mathbf{r} P=T_{1} \coprod T_{2}$. Then

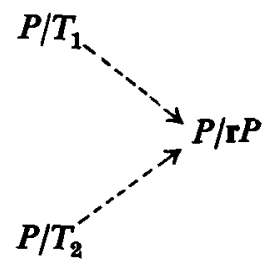

is part of $\mathrm{D}(P / \mathbf{r} P)$. Since there can be no nonzero composition of maps $P / T_{1} \longrightarrow$ $P / T_{2} \longrightarrow P / \mathrm{r} P$ or $P / T_{2} \longrightarrow P / T_{1} \longrightarrow P / \mathrm{r} P, \mathrm{D}(P / \mathrm{r} P)$ must be

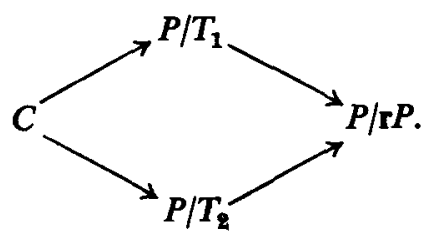

It is easy to see that if $h: X \rightarrow P / T_{1}$ is such that $X$ is indecomposable and $\underline{h}$ is not zero, then $h$ must be an epimorphism. Hence we get $\mathrm{D}\left(P / T_{1}\right)=C \rightarrow P / T_{1}$, and similarly $\mathrm{D}\left(P / T_{2}\right)=C \rightarrow P / T_{2}$. Considering (1), we see that this is a contradiction. Hence we conclude that $P$ is uniserial.

(d) Since $\Lambda$ and $\Lambda^{\prime}$ are stably equivalent, we know that $\Lambda^{o p}$ and $\Lambda^{\prime o p}$ are also stably equivalent [1]. Further, $\Lambda^{\prime o p}$ is Nakayama since $\Lambda^{\prime}$ is. Hence the indecomposable projective $\Lambda^{o p}$-modules satisfy (a), (b) and (c). We then use the ordinary duality for artin algebras to finish the proof of (d).

(e) This is a direct consequence of the earlier parts of the lemma.

We shall now go on to investigate the correspondence between the indecomposable nonprojective modules in $\bmod \Lambda$ and $\bmod \Lambda^{\prime}$ given by the stable equivalence.

LEMMA 2. Let $P_{1}$ be an indecomposable projective $\Lambda$-module which is not uniserial, and let $T_{1}=P_{1} / \mathbf{r} P_{1}$. Write $\mathbf{r} P_{1} / \mathbf{r}^{2} P_{1}=T_{2} \coprod T_{3}$, where $T_{2}$ and $T_{3}$ are nonisomorphic simple $\Lambda$-modules, and write $T_{4}=\operatorname{soc} P_{1}$. Then we have the following.

(a) $T_{1}$ corresponds to a $\Lambda^{\prime}$-module of length 2 , say $\left(\begin{array}{l}S_{1} \\ S_{2}\end{array}\right), P_{1} / \operatorname{soc} P_{1} \leftrightarrow\left(\begin{array}{c}S_{2} \\ S_{3}\end{array}\right)$, $\mathbf{r} P_{1} \leftrightarrow\left(\begin{array}{c}S_{3} \\ S_{4}\end{array}\right)$, and we can choose the numbering of $T_{2}$ and $T_{3}$ such that $T_{2} \leftrightarrow\left(\begin{array}{c}S_{2} \\ S_{3} \\ S_{4}\end{array}\right)$, $T_{3} \leftrightarrow S_{3},\left(\begin{array}{l}T_{1} \\ T_{2}\end{array}\right) \leftrightarrow S_{2}$ and $\left(\begin{array}{l}T_{1} \\ T_{3}\end{array}\right) \leftrightarrow\left(\begin{array}{l}S_{1} \\ S_{2} \\ S_{3}\end{array}\right)$. In particular, neither $T_{2}$ nor $T_{3}$ is isomorphic to $T_{1}$.

(b) $P_{2}$ is uniserial of length 3 and $P_{3}$ is uniserial of length 2 or 3. 
(c) $T_{4} \leftrightarrow\left(\begin{array}{c}S_{4} \\ S_{5}\end{array}\right)$ and $\left(\begin{array}{l}T_{2} \\ T_{4}\end{array}\right) \leftrightarrow S_{4}$. Hence $P_{4}$ has length 3 if it is uniserial.

(d) If $T_{5}=\operatorname{soc} P_{2}$ and $P_{4}$ is not uniserial, then $T_{5} \leftrightarrow\left(\begin{array}{c}S_{5} \\ S_{6} \\ S_{7}\end{array}\right)$.

(e) If $P_{3}$ has length 3 , then $P_{4}$ is not uniserial.

(f) $T_{2}$ is contained in no other indecomposable module of length 2 than $\left(\begin{array}{l}T_{1} \\ T_{2}\end{array}\right)$, $T_{3}$ in no other than $\left(\begin{array}{l}T_{1} \\ T_{3}\end{array}\right)$, and $T_{4}$ in no other than $\left(\begin{array}{l}T_{2} \\ T_{4}\end{array}\right)$ and $\left(\begin{array}{l}T_{3} \\ T_{4}\end{array}\right)$.

Proof. (a) It is not hard to see that we have the diagrams
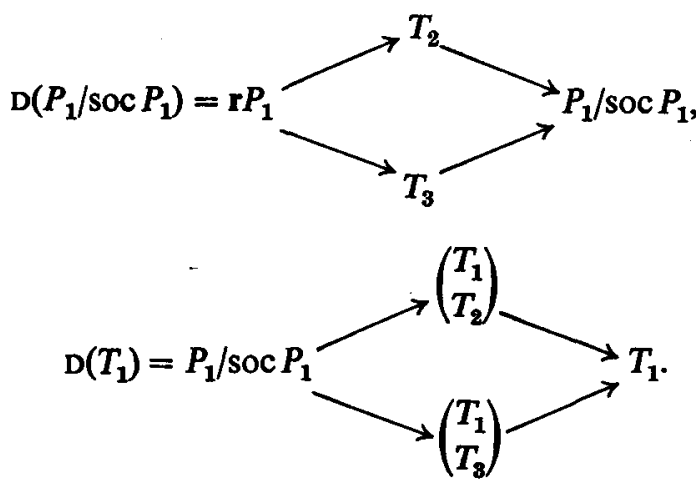

All claims in (a) follow by comparing this with the diagrams (1), (2) and (3) in Section 1.

have

(b) Since $T_{2} \leftrightarrow\left(\begin{array}{l}S_{2} \\ S_{3} \\ S_{4}\end{array}\right), P_{2}$ must be uniserial by (a). And by (1) we know that we

$$
\mathrm{D}\left(T_{2}\right)=A \rightarrow B \rightarrow T_{2} \text {. }
$$

Since we must have $A \leftrightarrow S_{4}, B \leftrightarrow\left(\begin{array}{l}S_{3} \\ S_{4}\end{array}\right)$, it is easy to see that there is no nonzero map modulo projectives from $A$ to an indecomposable module not in the diagram. Since $B$ is not simple, it then follows that $A / \mathbf{r} A$ is simple. We must then have $B \cong \mathbf{r} P_{1}$ and $A \cong\left(\begin{array}{l}T_{2} \\ T_{4}\end{array}\right)$. This shows that $P_{2}$ is uniserial of length 3 .

Since $T_{3} \leftrightarrow S_{3}$ and $r P_{1} \leftrightarrow\left(\begin{array}{l}S_{3} \\ S_{4}\end{array}\right)$, we have that $\mathrm{D}\left(T_{3}\right)$ is $C \rightarrow \mathrm{r} P_{1} \rightarrow T_{3}$ or $\mathbf{r} P_{1} \rightarrow T_{3}$. In the first case we must as above have that $C / \mathbf{r} C$ is simple. This shows that $P_{3}$ is uniserial of length 2 or 3. 
(c) If $P_{3}$ has length 3 , consider the diagram

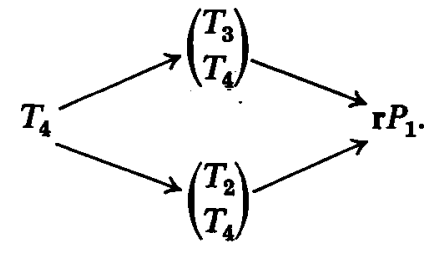

Since $\mathbf{r} P_{1} \leftrightarrow\left(\begin{array}{c}S_{3} \\ S_{4}\end{array}\right)$, we must have $T_{4} \leftrightarrow\left(\begin{array}{c}S_{4} \\ S_{5}\end{array}\right)$. Further, $\left(\begin{array}{l}T_{2} \\ T_{4}\end{array}\right)$ corresponds to either $S_{4}$ or $\left(\begin{array}{l}S_{4} \\ S_{5} \\ S_{6}\end{array}\right)$. Since there is a nonzero map from $\left(\begin{array}{l}T_{2} \\ T_{4}\end{array}\right)$ to $T_{2}$, and $T_{2} \leftrightarrow\left(\begin{array}{l}S_{2} \\ S_{3} \\ S_{4}\end{array}\right)$, we must have $\left(\begin{array}{l}T_{2} \\ T_{4}\end{array}\right) \leftrightarrow S_{4}$.

If $P_{3}$ has length 2, we have $\mathrm{D}\left(T_{3}\right)=\mathbf{r} P_{1} \rightarrow T_{3}$. Since the projective cover of $S_{3}$ must then have length 2 and $\mathrm{r} P_{1} \leftrightarrow\left(\begin{array}{l}S_{3} \\ S_{4}\end{array}\right)$, we get $\mathrm{D}\left(\mathrm{r} P_{1}\right)=\left(\begin{array}{l}T_{2} \\ T_{4}\end{array}\right) \rightarrow \mathrm{r} P_{1}$, and hence $\left(\begin{array}{l}T_{2} \\ T_{4}\end{array}\right) \leftrightarrow S_{4}$.

Considering $\mathrm{D}\left(T_{2}\right)$, we see that there is no nonprojective indecomposable module mapping onto $\left(\begin{array}{l}T_{2} \\ T_{4}\end{array}\right)$. Hence any nonzero map from an indecomposable nonprojective $\Lambda$-module not isomorphic to $\left(\begin{array}{l}T_{2} \\ T_{4}\end{array}\right)$ has $T_{4}$ as image. It follows that $T_{4} \leftrightarrow\left(\begin{array}{l}S_{4} \\ S_{5}\end{array}\right)$. Assume that $P_{4}$ is uniserial, and assume that

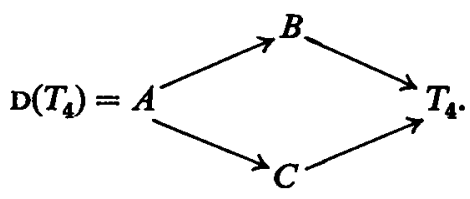

It is then not hard to see, and it is similar to our previous arguments, that $A / \mathrm{r} A$, $B / \mathrm{r} B, C / \mathrm{r} C$ would all be isomorphic to $T_{4}$, and $\mathrm{D}\left(T_{4}\right)$ could then not be as assumed. We must then have $\mathrm{D}\left(T_{4}\right)=\left(\begin{array}{l}T_{4} \\ T_{5}\end{array}\right) \rightarrow T_{4}$, so that $P_{4}$ has length 3 .

(d) Let $T_{5}=\operatorname{soc} P_{2}$ and assume that $P_{4}$ is not uniserial. We can then write $\mathbf{r} P_{4} / \mathbf{r}^{2} P_{4}=T_{5} \bigsqcup T_{6}$, and we know from (a) that $T_{5}$ and $T_{6}$ correspond to $S_{6}$ and $\left(\begin{array}{l}S_{5} \\ S_{6} \\ S_{7}\end{array}\right)$, and that $\left(\begin{array}{l}T_{4} \\ T_{5}\end{array}\right)$ and $\left(\begin{array}{l}T_{4} \\ T_{6}\end{array}\right)$ correspond to $S_{5}$ and $\left(\begin{array}{l}S_{4} \\ S_{5} \\ S_{6}\end{array}\right)$. There is some non- 
zero map modulo projectives $\left(\begin{array}{l}T_{4} \\ T_{6}\end{array}\right) \rightarrow\left(\begin{array}{l}T_{2} \\ T_{4}\end{array}\right)$, but no nonzero map $S_{5} \rightarrow S_{4}$. Hence we must have $\left(\begin{array}{l}T_{4} \\ T_{6}\end{array}\right) \leftrightarrow\left(\begin{array}{l}S_{4} \\ S_{5} \\ S_{6}\end{array}\right)$ and $\left(\begin{array}{l}T_{4} \\ T_{5}\end{array}\right) \leftrightarrow S_{5}$. It further follows that $T_{6} \leftrightarrow S_{6}$ and $T_{5} \leftrightarrow\left(\begin{array}{c}S_{5} \\ S_{6} \\ S_{7}\end{array}\right)$

(e) Assume that $P_{3}$ has length 3. We must then have

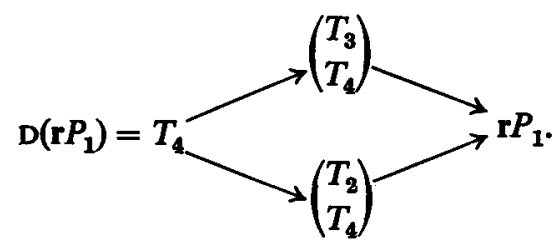

If $T_{4} \cong T_{1}, P_{4} \cong P_{1}$ is not uniserial. Assume then that $T_{4} \neq T_{1}$, and let $T_{6}=\operatorname{soc} P_{3}$. We then have that $\left(\begin{array}{l}T_{4} \\ T_{5}\end{array}\right)$ is contained in $P_{2}$ and $\left(\begin{array}{l}T_{4} \\ T_{6}\end{array}\right)$ in $P_{3}$. If $T_{5} \cong T_{6}$, then $P_{2}$ and $P_{3}$ would both be contained in the injective envelope of $T_{5}$, which is a contradiction to the structure of the indecomposable injective $\Lambda$-modules. Hence $T_{5}$ and $T_{6}$ are not isomorphic, so that $\left(\begin{array}{l}T_{4} \\ T_{5}\end{array}\right)$ and $\left(\begin{array}{l}T_{4} \\ T_{6}\end{array}\right)$ are not isomorphic. Hence $P_{4}$ is not uniserial.

(f) If $T_{2}$ is contained in another indecomposable uniserial $\Lambda$-module of length 2 than $\left(\begin{array}{l}T_{1} \\ T_{2}\end{array}\right)$, then $T_{2}$ would be contained in a nonuniserial indecomposable projective $\Lambda$-module, and $T_{2}$ would by (b) correspond to a uniserial $\Lambda^{\prime}$-module of length 2 , which is impossible by (a). The argument that $T_{3}$ is contained in no other uniserial module of length 2 than $\left(\begin{array}{l}T_{1} \\ T_{3}\end{array}\right)$ is analogous. $T_{4}$ is contained in no other uniserial modules of length 2 than $\left(\begin{array}{l}T_{2} \\ T_{4}\end{array}\right)$ and $\left(\begin{array}{l}T_{3} \\ T_{4}\end{array}\right)$, since the injective envelope of $T_{4}$ would otherwise not be of the desired type.

Lemma. 3. Assume $T_{i} \leftrightarrow\left(\begin{array}{c}S_{i} \\ S_{i+1}\end{array}\right), P_{i}$ uniserial, and let $T_{i+1}=\mathbf{r} P_{i} / \mathbf{r}^{2} P_{i}$.

(a) $P_{i}$ has length $3, T_{i+1} \leftrightarrow\left(\begin{array}{c}S_{i+1} \\ S_{i+2}\end{array}\right)$, and $\left(\begin{array}{c}T_{i} \\ T_{i+1}\end{array}\right)$ is the only uniserial module of length 2 in which $T_{t+1}$ is contained.

(b) If $P_{i+1}$ is not uniserial, then $T_{i+2}=\operatorname{soc} P_{i}$ corresponds to a uniserial $\Lambda^{\prime}$-module of length 3 . 
Proof. (a) Assume first that

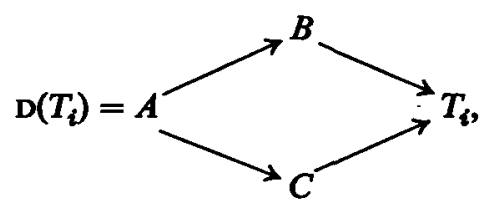

where $B \leftrightarrow S_{i+1}$ and $C \leftrightarrow\left(\begin{array}{c}S_{i} \\ S_{t+1} \\ S_{t+2}\end{array}\right)$. $C / \mathrm{r} C$ has only one copy of $T_{i}$. For otherwise there would be maps $u: C \rightarrow T_{i}, v: C \rightarrow T_{i}$ such that for any $t: T_{i} \rightarrow T_{i}, \underline{t} \underline{u} \neq \underline{v}$. If some simple $\Lambda$-module $T \neq T_{l}$ was a summand of $C / \mathrm{r} C$, we would have a nonzero $\underline{f}: \underline{C} \leftrightarrow \underline{T}$, hence $T \leftrightarrow S_{i}$. But since there is a nonzero map from $\left(\begin{array}{c}S_{l} \\ S_{l+1}\end{array}\right)$ to $\underline{S}_{i}$, but not from $\underline{T}_{i}$ to $\underline{T}$, we get a contradiction. Hence $C / \mathbf{r} C \cong T_{i}$. In a similar way we also prove that $A / \mathbf{r} A \cong T_{i}$ and $B / \mathbf{r} B \cong T_{i}$. But since $P_{i}$ is uniserial, we then get a contradiction to $\mathrm{D}\left(T_{t}\right)$ being of the above type. Since $T_{i} \leftrightarrow\left(\begin{array}{c}S_{l} \\ S_{i+1}\end{array}\right)$ we must consequently have $\mathrm{D}\left(T_{i}\right)=A \rightarrow T_{i}$. Similar to the above we can prove that $A / \mathrm{r} A$ is simple. This shows that $P_{l}$ has length 3 , and that $A \cong\left(\begin{array}{c}T_{i} \\ T_{i+1}\end{array}\right)$, where $T_{t+1} \cong \mathrm{r} P_{i} / \mathbf{r}^{2} P_{i}$. It then follows that $\left(\begin{array}{c}T_{i} \\ T_{i+1}\end{array}\right) \leftrightarrow S_{t+1}$. Since no indecomposable nonprojective $\Lambda$-module maps onto $\left(\begin{array}{c}T_{i} \\ T_{i+1}\end{array}\right)$, any nonzero map $X \rightarrow\left(\begin{array}{c}T_{i} \\ T_{i+1}\end{array}\right)$, where $X$ is indecomposable and not projective and not isomorphic to $\left(\begin{array}{c}T_{i} \\ T_{i+1}\end{array}\right)$, factors through $T_{i+1} \rightarrow\left(\begin{array}{c}T_{i} \\ T_{i+1}\end{array}\right)$. Hence $T_{i+1} \leftrightarrow\left(\begin{array}{c}S_{i+1} \\ S_{i+2}\end{array}\right)$.

(b) We assume now that $P_{i+1}$ is not uniserial, $\mathbf{r} P_{t+1} / \mathbf{r}^{2} P_{i+1}=T_{i+2} \coprod T_{i+3}$ such that $T_{i+2}=\operatorname{soc} P_{i}$.

We have seen that $\left(\begin{array}{c}T_{i} \\ T_{i+1}\end{array}\right) \leftrightarrow S_{i+1}$, hence we have

$$
\mathrm{D}\left(\left(\begin{array}{c}
T_{i} \\
T_{i+1}
\end{array}\right)\right)=\left(\begin{array}{c}
T_{i+1} \\
T_{i+3}
\end{array}\right) \rightarrow T_{i+1} \rightarrow\left(\begin{array}{c}
T_{i} \\
T_{i+1}
\end{array}\right),
$$

so that $\left(\begin{array}{l}T_{i+1} \\ T_{i+3}\end{array}\right) \leftrightarrow\left(\begin{array}{l}S_{i+1} \\ S_{i+2} \\ S_{i+3}\end{array}\right)$. By (a), $T_{i+2}$ then corresponds to $\Lambda^{\prime}$-module of length 3 .

We are now ready to finish the proof that the conditions of the Theorem are necessary. Let $\Lambda$ be an indecomposable algebra stably equivalent to a Nakayama algebra $\Lambda^{\prime}$ of Loewy length 4 , and assume that $\Lambda$ is not Nakayama. The first part 
follows from Lemma 1. Choose a simple $\Lambda$-module $T_{1}$ such that $P_{1}$ is not uniserial. By Lemma 1(b) we can write $\mathrm{r} P_{1} / \mathbf{r}^{2} P_{i}=T_{2} \bigsqcup T_{3}$, where $T_{2}$ and $T_{3}$ are not isomorphic simple modules, and we know by Lemma 2(a) that no two of the simple modules $T_{1}, T_{2}$ and $T_{3}$ are isomorphic and that we can choose $T_{2}$ such that it corresponds to a $\Lambda^{\prime}$-module of length 3 . We further know from Lemma 2(b) that $P_{2}$ is uniserial of length $3, P_{3}$ is uniserial of length 2 or 3 . If $P_{3}$ has length 3 , we know by Lemma 2(e) that $P_{4}$ is not uniserial, where $T_{4}=\operatorname{soc} P_{1}$. If $P_{3}$ has length 2 we know by Lemma 2(c) that $P_{4}$ is uniserial of length 3 or is not uniserial.

Assume that $P_{4}$ is uniserial. Since $T_{4}$ corresponds to a $\Lambda^{\prime}$-module of length 2 by Lemma 2(c), we have, for $T_{5}=\mathbf{r} P_{4} / \mathbf{r}^{2} P_{4}$, that $P_{5}$ is uniserial of length 3 or is not uniserial by Lemma 3 . In both cases $T_{5}$ corresponds to a $\Lambda^{\prime}$-module of length 2 by Lemma 2(a) and Lemma 3(a).

In general, if $P_{l}$ is not uniserial, we choose the numbering such that

$$
\mathbf{r} P_{i} / \mathbf{r}^{2} P_{i}=T_{i+1} \bigsqcup T_{i+2},
$$

where $T_{t+1}$ corresponds to a $\Lambda^{\prime}$-module of length $3, T_{i+3}=\operatorname{soc} P_{i}$. We know that $P_{i+1}$ and $P_{t+2}$ are uniserial and that if $P_{i+3}$ is not uniserial, then it is by Lemma 2(d) compatible with our choice of numbering that $T_{i+4}=\operatorname{soc} P_{i+1}$, and $T_{i+5}=$ soc $P_{t+2}$ if $P_{i+2}$ has length 3. If $P_{i+4}$ is uniserial, we know by Lemma 2(c) that $P_{i+4}$ has length 3 and that $T_{i+4}$ corresponds to a $\Lambda^{\prime}$-module of length 2 . So in general if $P_{j}$ is uniserial of length 3 and $T_{j}$ corresponds to a $\Lambda^{\prime}$-module of length 2 , we choose $T_{j+1}=\mathrm{r} P_{j} / \mathbf{r}^{2} P_{j}$ and $T_{j+2}=\operatorname{soc} P_{j}$. Since the $T_{i+1}$ and $T_{i+2}$ above do not correspond to a $\Lambda^{\prime}$-module of length 2 by Lemma 2(a), this is not in conflict with the above choice. Further, we know by Lemma 3(a) that $T_{j+1}$ corresponds to a $\Lambda^{\prime}$-module of length 2 and that $P_{J+1}$ is uniserial of length 3 or is not uniserial. If $P_{j+1}$ is not uniserial, we have by Lemma 3(b) that $T_{j+2}=\operatorname{soc} P_{j}$ corresponds to a $\Lambda^{\prime}$-module of length 3 , so that we have no conflict in our choice here.

We know that no two of the modules $T_{1}, T_{2}$ and $T_{3}$ are isomorphic. Let $T_{l}$ be such that $T_{i} \cong T_{J}, j<i$, and $i$ is smallest possible. Assume first that $j>1$. Then $T_{t}$ and $T_{j}$ are contained in the same uniserial modules of length 2 , either only

$$
\left(\begin{array}{c}
T_{i-1} \\
T_{i}
\end{array}\right) \text { or }\left(\begin{array}{c}
T_{i-1} \\
T_{i}
\end{array}\right) \text { and }\left(\begin{array}{c}
T_{t-2} \\
T_{i}
\end{array}\right)
$$

This will give a contradiction to the minimality of $i$, and hence $j=1$. This finishes the proof that our conditions are necessary.

\section{Sufficiency}

In this section we shall sketch a proof of the fact that the conditions of the main theorem are sufficient. So assume that $\Lambda$ is an indecomposable algebra satisfying the conditions of the theorem. It follows as a special case of a result of C. M. 
Ringel (unpublished) that each indecomposable $\Lambda$-module is a factor module of an indecomposable projective module or of the type $\mathrm{r} Q$, where $Q$ is indecomposable projective.

It is not hard to see that the endomorphism rings of the simple $\Lambda$-modules are all isomorphic to the same division ring $D$. Further, it is also not hard to see that for each indecomposable nonprojective $\Lambda$-module $M$, End $0 \cong \underline{M D}$, and if $N$ is also an indecomposable nonprojective $\Lambda$-module, then $\operatorname{Hom}(\underline{M}, \underline{N})$ is zero or isomorphic to $D$ as a two sided $D$-module.

Let $n$ be the number of simple nonisomorphic $\Lambda$-modules and let $\left(r_{1}, \ldots, r_{n}\right)$ be the following sequence of numbers, where each $r_{t}$ is either 3 or 4 . If $P_{t}$ is not uniserial, then let $r_{i}=r_{t+1}=4$, and let $r_{t+2}=3$ if $P_{i+2}$ has length $2, r_{t+2}=4$ if $P_{i+2}$ has length 3 . The $r_{j}$ which are not already defined by the above are defined to be 3. We then know that it is possible to find an indecomposable Nakayama algebra $\Lambda^{\prime}$ with indecomposable projective modules $Q_{1}, \ldots, Q_{n}$ such that

$$
\mathbf{r} Q_{i} / \mathbf{r}^{2} Q_{i} \cong Q_{i+1} / \mathbf{r} Q_{i+1}
$$

for $i=1, \ldots, n$, where $Q_{n+1}=Q_{1}, L\left(Q_{i}\right)=r_{i}$ and the endomorphism ring of each simple $\Lambda^{\prime}$-module is isomorphic to $D$ (see Kupisch, 1959 and Murase, 1964). $\left(r_{1}, \ldots, r_{n}\right)$ is then said to be an admissible sequence associated with $\Lambda^{\prime}$, and it is unique up to cyclic permutation.

To finish our proof it is then sufficient to show that $\Lambda$ is stably equivalent to the $\Lambda^{\prime}$ chosen above. Since $n \geqslant 3$, we have that End $(\underline{A}) \cong D$ for each indecomposable nonprojective $\Lambda^{\prime}$-module $A$, and that $\operatorname{Hom}(\underline{A}, \underline{B})$ is zero or isomorphic to $D$ as a two sided $D$-module if $A$ and $B$ are indecomposable nonprojective $\Lambda^{\prime}$-modules. By the above remarks about $\Lambda$, it will then suffice to define a correspondence $F$ between the indecomposable nonprojective modules over $\Lambda$ and $\Lambda^{\prime}$, which has the following properties. If $A, B$ and $C$ are indecomposable nonprojective $\Lambda$-modules, then $\operatorname{Hom}(\underline{A}, \underline{B}) \neq 0$ if and only if $\operatorname{Hom}(\underline{F} \underline{A}, \underline{F} \underline{B}) \neq 0$. And if $f: A \rightarrow B, \mathrm{~g}: B \rightarrow C$, $f^{\prime}: F A \rightarrow F B, g^{\prime}: F B \rightarrow F C$ are such that $\underline{f}, \underline{g}, \underline{f}^{\prime}, \underline{g^{\prime}}$ are not zero, then $\underline{g} \underline{f}$ is zero if and only if $\underline{g}^{\prime} \underline{f}^{\prime}$ is zero.

We shall explain the correspondence $F$ below. It is not hard to check that the desired properties hold, so that $F$ can be extended to an equivalence $F: \underline{\bmod \Lambda \rightarrow}$ $\underline{\bmod \Lambda^{\prime}}$.

(1) Assume that $P_{i}$ is not uniserial. Then we define

$$
\begin{gathered}
T_{i} \rightarrow\left(\begin{array}{c}
S_{t} \\
S_{t+1}
\end{array}\right), \quad T_{t+1} \rightarrow\left(\begin{array}{c}
S_{t+1} \\
S_{t+2} \\
S_{t+3}
\end{array}\right), \\
T_{t+2} \rightarrow S_{t+2}, \quad\left(\begin{array}{c}
T_{t} \\
T_{t+1}
\end{array}\right) \rightarrow S_{t+1},
\end{gathered}
$$




$$
\begin{gathered}
\left(\begin{array}{c}
T_{i} \\
T_{i+2}
\end{array}\right) \rightarrow\left(\begin{array}{c}
S_{i} \\
S_{i+1} \\
S_{i+2}
\end{array}\right), \quad\left(\begin{array}{c}
T_{i+1} \\
T_{i+3}
\end{array}\right) \rightarrow S_{i+3}, \\
P_{i} / \operatorname{soc} P_{i} \rightarrow\left(\begin{array}{c}
S_{i+1} \\
S_{i+2}
\end{array}\right) \text { and } \mathbf{r} P_{i} \rightarrow\left(\begin{array}{c}
S_{i+2} \\
S_{i+3}
\end{array}\right) .
\end{gathered}
$$

If $P_{t+2}$ has length 3 , then $\left(\begin{array}{l}T_{t+2} \\ T_{t+3}\end{array}\right)$ is also not projective. In this case we also have $r_{i+2}=4$ and so we define

$$
\left(\begin{array}{c}
T_{i+2} \\
T_{i+3}
\end{array}\right) \rightarrow\left(\begin{array}{c}
S_{i+2} \\
S_{i+3} \\
S_{i+4}
\end{array}\right)
$$

(2) Assume that $P_{j}$ is uniserial of length 3 and that $T_{j}$ is not a submodule of any $P / \operatorname{soc} P$, for a non uniserial indecomposable projective $\Lambda$-module $P$. Then,

$$
T_{j} \rightarrow\left(\begin{array}{c}
S_{j} \\
S_{j+1}
\end{array}\right) \text { and }\left(\begin{array}{c}
T_{j} \\
T_{j+1}
\end{array}\right) \rightarrow S_{j+1}
$$

We end this section with some comments to our theorem.

(i) It follows from our theorem that the structure of the indecomposable non Nakayama algebras $\Lambda$ stably equivalent to a Nakayama algebra $\Lambda^{\prime}$ of Loewy length at most 4 is given by the following data. Let $\alpha$ denote a triple $\left(P_{1}, P_{i+1}, P_{i+2}\right)$ of indecomposable projective $\Lambda$-modules, where $P_{i}$ is not uniserial, $P_{i+1}$ and $P_{i+2}$ are uniserial of length 3 . Let $\beta$ denote a triple $\left(P_{i}, P_{i+1}, P_{i+2}\right)$, where $P_{i}$ is not uniserial, $P_{i+1}$ is uniserial of length 3 and $P_{i+2}$ is uniserial of length 2 . Let $\gamma$ denote $\left(P_{i}\right)$, where $P_{t}$ is uniserial of length 3 and $P_{i} / \mathbf{r} P_{i}$ is not a summand of any $\mathbf{r} P / \mathbf{r}^{2} P$ for an indecomposable nonuniserial projective $\Lambda$-module $P$. To each $\alpha$ corresponds the part of the admissible sequence for $\Lambda^{\prime}(4,4,4)$, to $\beta(4,4,3)$ and to $\gamma(3)$. So the structure of $\Lambda$ is given by a cycle of $\alpha^{\prime} s, \beta^{\prime}$ s and $\gamma^{\prime}$, with the requirement that no $\gamma$ follows an $\alpha$.

(ii) Let $\Lambda$ be stably equivalent to an indecomposable Nakayama algebra $\Lambda^{\prime}$ of Loewy length 4 . If some maximal number of consecutive 4 in the admissible sequence for $\Lambda^{\prime}$ is $3 i+1$, then by the above $\Lambda$ must be Nakayama. Further it is easy to see that $\Lambda^{\prime}$ is selfinjective if and only if all entries in the admissible sequence are 4. Hence it also follows from the above that if $\Lambda^{\prime}$ is selfinjective and the number of simple $\Lambda^{\prime}$-modules is not divisible by 3 , then $\Lambda$ is Nakayama. And if $\Lambda^{\prime}$ is not selfinjective and some maximal number of consecutive 4 in the admissible sequence for $\Lambda^{\prime}$ is not $3 i+2$, then $\Lambda$ is Nakayama.

\section{References}

M. Auslander and I. Reiten (1973), 'Stable equivalence of artin algebras', Proc. Conf. on orders, group rings and related topics, pp. 8-64 (Lecture Notes in Mathematics 353, Springer Verlag, New York). 
M. Auslander and I. Reiten (1975), 'Stable equivalence of dualizing $R$-varieties III: Dualizing $R$-varieties stably equivalent to hereditary dualizing $R$-varieties', $A d v$. in Math. 17, 122-142.

M. Auslander and I. Reiten (1977a), 'Representation theory of artin algebras IV: Invariants given by almost split sequences', Comm. in Algebra 5 (5), 443-518.

M. Auslander and I. Reiten (1977b), 'Representation theory of artin algebras V: Methods for computing almost split sequences and irreducible morphisms', Comm. in Algebra 5 (5), 519-554.

H. Kupisch (1959), Beiträge zur Theorie nichthalbeinfacher Ringe mit Minimalbedingung', J. Reine Angew. Math. 201, 100-112.

I. Murase (1964), 'On the structure of generalized uniserial rings III', Sci. Pap. Coll. Gen. Educ. Univ. Tokyo 14, 11-25.

I. Reiten (1975), 'Stable equivalence of dualizing $R$-varieties VI: Nakayama dualizing $R$-varieties', Adv. in Math. 17, 196-211.

I. Reiten (1976), 'Stable equivalence of self injective algebras', J. of Algebra 14 (1), 63-74.

I. Reiten (1977), 'Correction to my paper on Nakayama $R$-varieties', Adv. in Math. 23, 211-212.

I. Reiten (1978), 'A note on stable equivalence and Nakayama algebras', Proc. Amer. Math. Soc. (to appear).

Department of Mathematics

University of Trondheim, NLHT

7000 Trondheim

Norway 